\title{
Impact of CT attenuation correction on the viability pattern assessed by (99m)Tc-tetrofosmin SPECT/ (18)F-FDG PET
}

\author{
Nkoulou, R ; Pazhenkottil, A P ; Buechel, R R ; Husmann, L ; Valenta, I ; Herzog, B A ; Wolfrum, M ;
} Ghadri, J R ; Kaufmann, P A

\begin{abstract}
SPECT myocardial perfusion imaging (MPI) is commonly used for comprehensive interpretation of metabolic PET FDG imaging in ischemic dysfunctional myocardium. We evaluated the difference in scan interpretation introduced by CT attenuation correction (CTAC) of SPECT MPI in patients undergoing viability characterization by $(99 \mathrm{~m})$ Tc SPECT MPI/PET FDG. In 46 consecutive patients (mean age 64, range 36-83 years) with dysfunctional myocardium, we analyzed viability from combined SPECT MPI and PET FDG scanning without attenuation correction (NC) and with CTAC for SPECT MPI. FDG uptake was classified in groups of percent uptake using the segment with maximum tracer in SPECT perfusion uptake as reference. Viability patterns were categorized as normal, mismatch, mild match and scar by relative comparison of SPECT and PET. Applying CTAC introduced a different reference segment for the normalization of PET FDG study in $57 \%$ of cases. As a result, the flow-metabolism pattern changed in $28 \%$ of segments, yielding a normal, mismatch, mild match and scar pattern in 462 , 150,123 , and 47 segments with $\mathrm{NC}$ and 553, 86, 108, and 35 with CTAC, respectively $(\mathrm{P}=0.001)$. Thus, by introducing CTAC for SPECT MPI $25 \%$ of segments originally classified as scar were reclassified and the number of normal segments increased by $20 \%$. Introducing CTAC decreased by $54 \%$ the number of patients with possible indication for revascularization, from $26 / 46$ to $12 / 46(\mathrm{P}<0.001)$. Different interpretation of myocardial viability can be observed when using CTAC instead of NC SPECT MPI as reference for PET FDG scans.
\end{abstract}

DOI: https://doi.org/10.1007/s10554-010-9719-8

Posted at the Zurich Open Repository and Archive, University of Zurich

ZORA URL: https://doi.org/10.5167/uzh-45499

Journal Article

Published Version

Originally published at:

Nkoulou, R; Pazhenkottil, A P; Buechel, R R; Husmann, L; Valenta, I; Herzog, B A; Wolfrum, M; Ghadri, J R; Kaufmann, P A (2011). Impact of CT attenuation correction on the viability pattern assessed by $(99 \mathrm{~m})$ Tc-tetrofosmin SPECT/ (18)F-FDG PET. International Journal of Cardiovascular Imaging, 27(6):913-921.

DOI: https://doi.org/10.1007/s10554-010-9719-8 


\title{
Impact of $\mathrm{CT}$ attenuation correction on the viability pattern assessed by ${ }^{99 m}$ Tc-tetrofosmin SPECT $/{ }^{18}$ F-FDG PET
}

\author{
Rene Nkoulou • Aju P. Pazhenkottil • Ronny R. Buechel • Lars Husmann • \\ Ines Valenta • Bernhard A. Herzog • Mathias Wolfrum • Jelena R. Ghadri • \\ Philipp A. Kaufmann
}

Received: 3 September 2010/Accepted: 23 September 2010/Published online: 8 October 2010

(C) Springer Science+Business Media, B.V. 2010

\begin{abstract}
SPECT myocardial perfusion imaging (MPI) is commonly used for comprehensive interpretation of metabolic PET FDG imaging in ischemic dysfunctional myocardium. We evaluated the difference in scan interpretation introduced by CT attenuation correction (CTAC) of SPECT MPI in patients undergoing viability characterization by ${ }^{99} \mathrm{~m} \mathrm{Tc}$ SPECT MPI/PET FDG. In 46 consecutive patients (mean age 64, range 36-83 years) with dysfunctional myocardium, we analyzed viability from combined SPECT MPI and PET FDG scanning without attenuation correction (NC) and with CTAC for SPECT MPI. FDG uptake was classified in groups of percent uptake using the segment with maximum tracer in SPECT perfusion uptake as reference. Viability patterns were categorized as normal, mismatch, mild match and scar by relative comparison of SPECT and PET. Applying CTAC introduced a different reference segment for the normalization of PET FDG study in $57 \%$ of cases. As a result, the flow-metabolism pattern changed in $28 \%$ of segments, yielding a
\end{abstract}

R. Nkoulou · A. P. Pazhenkottil · R. R. Buechel ·

L. Husmann · I. Valenta · B. A. Herzog ·

M. Wolfrum · J. R. Ghadri · P. A. Kaufmann ( $₫)$

Cardiac Imaging, University Hospital Zurich,

Rämistrasse 100, 8091 Zurich, Switzerland

e-mail: pak@usz.ch

P. A. Kaufmann

Zurich Center for Integrative Human Physiology (ZIHP),

University of Zurich, Zurich, Switzerland normal, mismatch, mild match and scar pattern in 462 , 150,123 , and 47 segments with NC and 553, 86, 108, and 35 with CTAC, respectively $(P=0.001)$. Thus, by introducing CTAC for SPECT MPI $25 \%$ of segments originally classified as scar were reclassified and the number of normal segments increased by 20\%. Introducing CTAC decreased by $54 \%$ the number of patients with possible indication for revascularization, from $26 / 46$ to $12 / 46(P<0.001)$. Different interpretation of myocardial viability can be observed when using CTAC instead of NC SPECT MPI as reference for PET FDG scans.

Keywords SPECT - CT attenuation correction Viability

\section{Introduction}

Due to the wide interindividual as well as regional variations in myocardial glucose uptake, accurate assessment of viability in ischemic dysfunctional left ventricular segments by ${ }^{18} \mathrm{~F}$-fluorodesoxyglucose (FDG) requires normalization of FDG using myocardial perfusion imaging (MPI) as relative standard of reference. This is particularly true when the concept of flow-metabolism "mismatch" is used where segments with decreased flow but preserved viability reveal hibernating myocardium. Among different techniques to evaluate myocardial perfusion, positron 
emission tomography (PET) is generally accepted as gold standard, providing quantitative measures of flow [1]. However, its availability has remained confined to centers with on-site cyclotron or with high patient volume throughput justifying the use of 82Rb-generators [2]. By contrast, single photon emission computer tomography (SPECT) MPI is widely available and compares well with PET MPI in association with metabolic PET FDG for the prediction of functional recovery. The fact that SPECT MPI is affected by non-uniform soft tissue attenuation has for long time been a caveat and a disadvantage compared to PET. Over the past years, introduction of methods for attenuation correction (AC) have substantially improved the accuracy of SPECT MPI, particularly when using computer tomography (CT) to obtain attenuation maps [3]. The added value of CTAC on the accuracy of SPECT MPI has been well established [4]. However, its impact on the interpretation of metabolic activity and hence on the final evaluation of viability studies, when using SPECT MPI as reference for FDG normalization, has not been systematically assessed. In view of the growing burden of coronary artery disease (CAD) related cardiac failure and the increased availability and use of SPECT devices with CTAC for MPI studies, a systematic evaluation of the impact of CTAC on viability interpretation seems pertinent. As prognostic data regarding appreciation of cardiac viability by nuclear techniques greatly relies on studies with no correction of attenuation (NC), our objective was to compare SPECT MPI with CTAC versus SPECT MPI without $\mathrm{AC}$ in the evaluation of metabolic activity assessed by PET FDG and the impact of introducing CTAC on the final definition of viability pattern.

\section{Materials and methods}

\section{Patient selection}

The study population consisted in 50 consecutive patients with angiographically proven $\mathrm{CAD}$ and decreased global left ventricular ejection fraction referred for an evaluation of viability by SPECT MPI/ PET FDG. The study was approved by the local ethics committee and patients gave their written informed consent.
CT imaging for attenuation correction

Attenuation correction scan covering the entire heart using prospectively ECG-triggered sequential images at $70 \%$ of the R-R interval was obtained on a LightSPeed VCT scanner (General Electric Medical Systems, Milwaukee, WI, USA) during deep inspiration. $2.5 \mathrm{~mm}$ section thickness, a $0.35 \mathrm{~s}$ gantry rotation time at $120 \mathrm{kV}$ and $200-250 \mathrm{~mA}$, depending on the patient's size were used as scanning parameters. CT images were reconstructed at a $5.0 \mathrm{~mm}$ section thickness by using a reconstruction algorithm with a $512 \times 512$ matrix and a full-chest-size adapted FOV of $50 \times 50 \mathrm{~cm}$. These reconstructed images were used both for attenuation correction and for coronary calcium scoring as previously described [5].

Myocardial perfusion image acquisition and processing

All patients underwent a 1-day ECG-gated adenosine stress/rest or rest-only protocol. Post-stress image acquisition was performed $90 \mathrm{~min}$ after administration of $250-350 \mathrm{MBq}$ of ${ }^{99 \mathrm{~m}} \mathrm{Tc}$-tetrofosmin injected $3 \mathrm{~min}$ into the pharmacologic stress. Rest images acquisition was performed $4 \mathrm{~h}$ later after administration of $750-1050 \mathrm{MBq}$ of ${ }^{99 \mathrm{~m}} \mathrm{Tc}$-tetrofosmin. For rest-only protocol, images were acquired $90 \mathrm{~min}$ after administration of $250-350 \mathrm{MBq}$ of ${ }^{99 \mathrm{~m}} \mathrm{Tc}$-tetrofosmin. Gated SPECT studies were performed with a dual-head detector camera (Millenium VG and Hawkeye; GE Healthcare) as previously described [6]: a low-energy, high-resolution collimator; a $20 \%$ symmetric window at $140 \mathrm{keV}$; a $64 \times 64$ matrix; an elliptic orbit with step-and shoot acquisition at $3^{\circ}$ intervals over $180^{\circ}$; and a $20 \mathrm{~s}$ dwell time per stop. Acquisitions were gated at 16 frames per R-R cycle with a $50 \%$ window of acceptance.

For all patients, the summed ungated SPECT image set was reconstructed on a dedicated workstation (eNTEGRA or Xeleris; GE Medical systems, Milwaukee, Wis) by using an iterative reconstruction ordered-subset expectation maximization (OSEM, with 2 iterations and 10 subsets) and with CTAC correction. Polar maps of perfusion, wall motion, and wall thickening were derived by using a commercially available software package (Cedars QGS/QPS; Cedars-Sinai Medical Center, Los Angeles, Calif). 
PET FDG image acquisition and processing

Myocardial viability testing was performed on a PET/ CT scanner using FDG after completing the SPECT MPI. This sequence was chosen in order to avoid spill over from high-energy photons of ${ }^{18} \mathrm{~F}$ during SPECT acquisition. All patients were advised to fast for at least $6 \mathrm{~h}$ before the PET examination. The injection of labelled FDG was performed after a standardized oral glucose load [7]. Following injection of $250 \mathrm{MBq}$ of FDG, patients rested for about $1 \mathrm{~h}$ before image acquisition. Images were acquired on a GE Advance PET scanner or on a Discovery (LS/RX) PET/CT scanner (both GE Healthcare, Miwaukee, Wis). CT attenuation maps were used for AC as previously reported [8]. Acquired images were reconstructed using attenuation weighted-OSEM iterative reconstruction (2 iterations and 8 subsets). Fourier rebinning was used for the reduction of $3 \mathrm{D}$ data set into $2 \mathrm{D}$ equivalent [9]. FDG uptake polar maps were equally derived by using the Cedars QPS/QGS software package.

\section{SPECT MPI interpretation}

All polar maps were arranged in 17 segments then divided into coronary territories (LAD, left anterior descending; CX, left circumflex; RCA, right coronary artery) according to standard segmentation of left ventricle acknowledged by ACC/AHA [10]. All MPI scans were evaluated twice, i.e. NC and CTAC. A segment with an average uptake in MPI below $70 \%$ and below $50 \%$ of maximal uptake was classified as perfusion defect and severe perfusion defect, respectively [11].

\section{PET FDG scan interpretation}

The segment with maximum uptake on MPI scan was also considered to represent normal value for FDG polar map and FDG segmental uptake on this segment was set as $100 \%$ to normalize others accordingly. All segments were categorized according to the respective relative metabolic uptake into group A-E (group A- 0-24\% uptake; B- 25-49\%; C50-74\%; D- 75-99\%, E- $\geq 100$ ).

Interpretation of viability pattern

Viability was evaluated from integrating SPECT MPI and PET FDG scan results. Myocardial segments were categorized as normal (perfusion over $70 \%$ of SPECT maximum uptake), mismatch (perfusion $<70 \%$ and difference between FDG and MPI $>20 \%$ ), mild match (perfusion between 50 and $70 \%$ and difference between FDG and MPI uptake $<20 \%$ ) and scar (perfusion $<50 \%$ and difference between FDG and MPI uptake $<20 \%$ ) as previously proposed [12, 13]. Mismatch was considered to indicate jeopardized but viable myocardium.

Correlative invasive coronary angiography (ICA)

Where available, results from recent ICA (less than 6 month) and gated SPECT were integrated in the clinical evaluation of discordant cases. Coronary luminal narrowing were described as non-significant $(<50 \%$ stenosis in diameter), intermediate $(50-70 \%)$ or severe stenosis $(>70 \%)$.

Statistical analysis

Values were given as mean \pm standard deviation (SD). Bland-Altman limits of agreement and systematic bias were provided on the difference in tracer uptake after the SPECT MPI. Data were compared for difference in distribution and agreement using chi square and kappa $(\kappa)$ statistics. We considered $\mathrm{k}$ values of 0.4 , of $0.4-0.75$, and of $>0.75$ to represent poor, moderate to good, and excellent agreement, respectively. Mc Nemar test was used to compare difference in evaluation of patients with preserved viability. A two-tailed $P$ value under 0.05 was judged significant.

\section{Results}

Of the 50 patients, 8 had diabetes mellitus. Four of them presented an FDG study of poor quality with unability to compute an activity polar map. They were excluded from further analysis. The remaining 46 patients were considered for comparative study. Baseline characteristics of the study population are summarized in Table 1.

\section{SPECT MPI}

782 segments and 144 coronary territories were amenable to analysis. When comparing ${ }^{99 \mathrm{~m}}$ Tc-tetrofosmin uptake from NC versus CTAC, Bland-Altman 
Table 1 Characteristics of the study population

\begin{tabular}{ll}
\hline Number of patients & 46 \\
\hline Age, mean (range) & $64(36-83)$ \\
Gender, (F/M) & $5 / 41$ \\
BMI, mean (SD) & $26.3(3.59)$ \\
Diabetes & $9 \%$ \\
LVEF\%, mean (SD) & $33 \pm 15.95$ \\
ECG & \\
Normal & $19 \%$ \\
LBBB & $25 \%$ \\
Q waves & $32 \%$ \\
Symptoms & \\
Dyspnoea & $64 \%$ \\
Typical chest pain & $26 \%$ \\
Atypical chest pain & $17 \%$ \\
History of CAD & \\
Myocardial infarction & $44 \%$ \\
PCI & $62 \%$ \\
CABG & $13 \%$ \\
\hline BMI Body mass ind $\left(\mathrm{kg} / \mathrm{m}^{2}\right), L V E F$ & \\
\hline
\end{tabular}

BMI Body mass index $\left(\mathrm{kg} / \mathrm{m}^{2}\right), L V E F$ left ventricular ejection fraction, $L B B B$ left bundle branch block, $C A D$ coronary artery disease, $P C I$ percutaneous coronary intervention, $C A B G$ coronary artery bypass grafting

limits of agreement and the mean difference in uptake (CTAC minus NC) were comparable for LAD $(-12$ to $12 \% ; 0.6 \%)$ and $\mathrm{CX}(-11$ to $15 \% ; 2.1 \%)$, but markedly wider and the difference larger for RCA territory $(-12$ to $25 \% ; 5.9 \%)$. On the NC SPECT study, the segment with maximum uptake was in the CX (32/46) or the LAD (14/46) territory, while it was located in CX (27/46), LAD (9/46) and RCA territory (9/46) with CTAC SPECT. NC and CTAC study agreed on the segment with maximum uptake in 20 patients $(43 \%)$, while they differed on segments of the same coronary region in 11 patients $(24 \%)$ and of another coronary region in 15 patients (33\%) Fig. 1. On NC SPECT MPI there were 320 segments with less than $70 \%$ and 108 segments with less than $50 \%$ tracer uptake. After CTAC there were 228 and 55 segments with less than 70 and $50 \%$ uptake, respectively $(P<0.001)$. Accordingly, agreement between SPECT MPI with NC and after CTAC was moderate $(570 / 782 ; \quad 73 \%, \quad \kappa=0.47 ; \quad 95 \%$ CI $0.409-0.531$; weighted $\kappa=0.558)$.

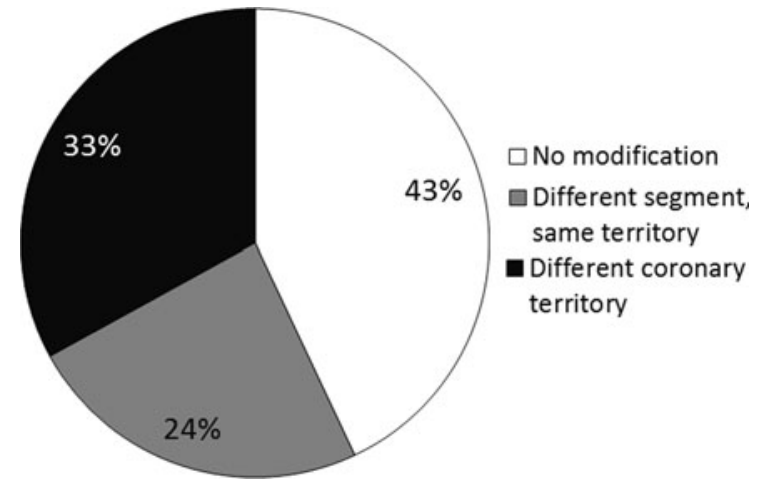

Fig. 1 Observed changes when using CTAC in the localisation of the segment with highest uptake on SPECT MPI (i.e. the reference segment) used for the evaluation of FDG uptake

\section{PET FDG}

Based on NC SPECT reference segments of maximum uptake, PET metabolic activity according to group A, B, C, D, and E was found in 6, 57, 185, 298, and 236 segments. After CTAC of SPECT the respective numbers were $6,58,157,295$, and 266 $(P=\mathrm{ns})$. The agreement in FDG classification between NC and CTAC reference was good $(79 \%$; $\kappa=0.70$, 95\%CI 0.66-0.74; weighted $\kappa=0.77$ ).

Table 2 provides the classification of segments with regard to FDG uptake according to the coronary territory with $\mathrm{NC}$ and after CTAC. Within vessel

Table 2 Distribution of segments according to FDG uptake

\begin{tabular}{llllll}
\hline Territory & \multicolumn{4}{l}{ FDG uptake } \\
\cline { 2 - 6 } & $\mathrm{A}(\%)$ & $\mathrm{B}(\%)$ & $\mathrm{C}(\%)$ & $\mathrm{D}(\%)$ & $\mathrm{E}(\%)$ \\
\hline LAD & & & & & \\
NC & 1 & 8 & 23 & 41 & 27 \\
CTAC & 1 & 8 & 21 & 38 & 31 \\
CX & & & & & \\
NC & 1 & 4 & 17 & 35 & 42 \\
CTAC & 1 & 5 & 13 & 31 & 49 \\
RCA & & & & & \\
NC & 1 & 9 & 31 & 36 & 23 \\
CTAC & 1 & 9 & 25 & 43 & 21 \\
\hline
\end{tabular}

Groups of FDG uptake: A (uptake 0-24\%), B (uptake 25-49\%), C (uptake 50-74\%), D (uptake 75-99\%), E (uptake $\geq 100 \%$ ), $L A D$ left anterior descending artery, $C X$ left circumflex artery, $R C A$ right coronary artery, $N C$ no attenuation correction, CTAC CT attenuation correction 
territories, there was no significant change from NC to CTAC $(P=\mathrm{ns})$. Consequently, there was a fairly good agreement between NC and CTAC in the LAD (77\% of agreement; $\kappa=0.685)$, as well as in the LCX $(71 \%, \kappa=0.6$; 95\%CI $0.518-0.683)$ and RCA $(75 \%, \kappa=0.662 ; 95 \%$ CI $0.585-0.739)$.

Flow-metabolism patterns

Using NC SPECT as reference there were 462, 150, 123, 47 segments with normal, mismatch, mild match or scar pattern. The respective numbers after CTAC were 553, 86, 108, $35(P=0.0001)$ Fig. 2 . These results were concordant in $566 / 782$ segments $(72 \%$, $\kappa=0.485 ; \quad 95 \%$ CI $\quad 0.427-0.544, \quad$ weighted $\kappa=0.557)$ showing moderate agreement between evaluation using NC versus CTAC SPECT MPI. In fact, after introducing CTAC, $25 \%$ of segments originally classified as scar were reclassified and the number of normal segments increased by $20 \%$. An example of the changes in flow-metabolism pattern introduced with the use of CTAC is given in Fig. 3.

The analysis in the LAD territory resulted in normal, mismatch, mild match or scar pattern in 214 , 43, 45, 20 segments with $\mathrm{NC}$ and 211, 45, 44, 22 segments with CTAC $(P=\mathrm{ns})$. The respective results in the $\mathrm{CX}$ territory were $170,24,29,7$ segments for NC and 190, 11, 27, 2 segments after CTAC $(P=n s)$. Finally, the respective numbers in the RCA territory were $78,83,49,20$ for $\mathrm{NC}$ and $152,30,37,11$ segments after CTAC $(P=0.0001)$ Table 3.

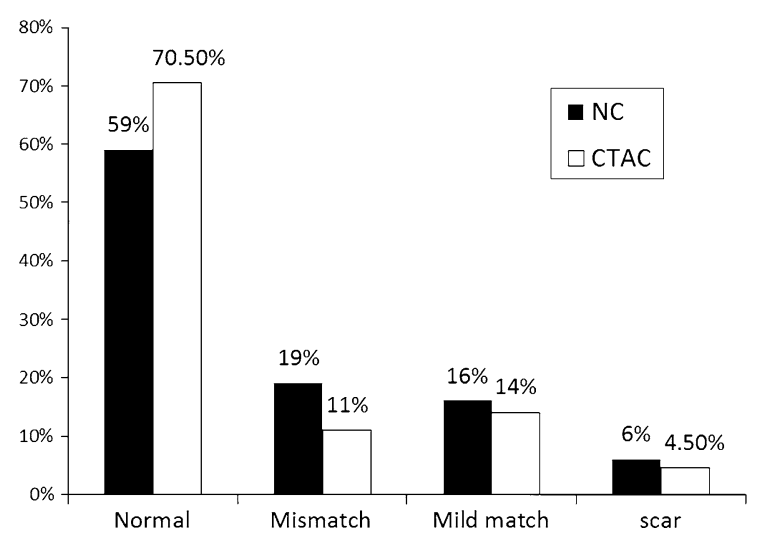

Fig. 2 Patterns of myocardial viability evaluated by SPECT MPI/PET FDG using NC and CTAC
The agreement between $\mathrm{NC}$ and CTAC with regard to viability patterns was good for the LAD segments $\quad(266 / 322, \quad 82 \%, \quad \kappa=0.667 ; \quad 95 \% \mathrm{CI}$ $0.588-0.746$, weighted $\kappa=0.722)$, moderate for the CX segments $(188 / 230,81 \%, \kappa=0.506 ; 95 \% \mathrm{CI}$ $0.370-0.641$, weighted $\kappa=0.583$ ) and fair for the RCA segments $(112 / 230,48 \%, \quad \kappa=0.257$; $95 \%$ C23.3I 0.163-0.350; weighted $\kappa=0.320$ ).

After complete evaluation using NC, 26/46 patients presented 3 or more viable segments (which represents at least $18 \%$ of the left ventricle). 19/46 patients presented 21 vessel territories with at least 3 viable segments. These were within the LAD $(n=3), \mathrm{CX}$ $(n=3)$ and RCA $(n=15)$ vessel territories, respectively. Of them, 2 patients had viable tissue in two vessel territories while the 17 remaining had viable tissue localized in one vessel territory.

By contrast, using CTAC there were 12/46 patients with 3 or more viable segments and 10/46 patients $(P=0.001)$. Eleven vessel territories presented at least 3 viable segments within the LAD $(n=5), \mathrm{CX}$ $(n=2)$ and RCA $(n=4)$ patients territory. Of them, 1 patient had viable tissue in two vessel territories and 9 in one vessel territory. As patients presenting at least 3 viable or ischemic segments are generally considered good candidates for revascularization, introducing CTAC decreased the number of patients with possible indication for revascularization by $54 \%$, from $26 / 46$ to $12 / 46(P<0.001)$.

\section{Correlative ICA}

A recent ICA was available in 33 study patients. This includes 10 of the 14 patients with discordant viability patterns between $\mathrm{NC}$ and CTAC. Ten patients with ICA presented an indication for revascularization (mismatch pattern) by using $\mathrm{NC}$ but normal pattern when applying CTAC, all in the RCA territory. The ICA largely supported the CTAC findings, as 4 angiographies presented no coronary lesions, 2 had an intermediate stenosis and only 4 had a severe stenosis which was however collateralized (grade III according to Rentrop [14]).

\section{Discussion}

Our study is the first to report on the impact of the recently introduced CTAC for SPECT MPI on the 

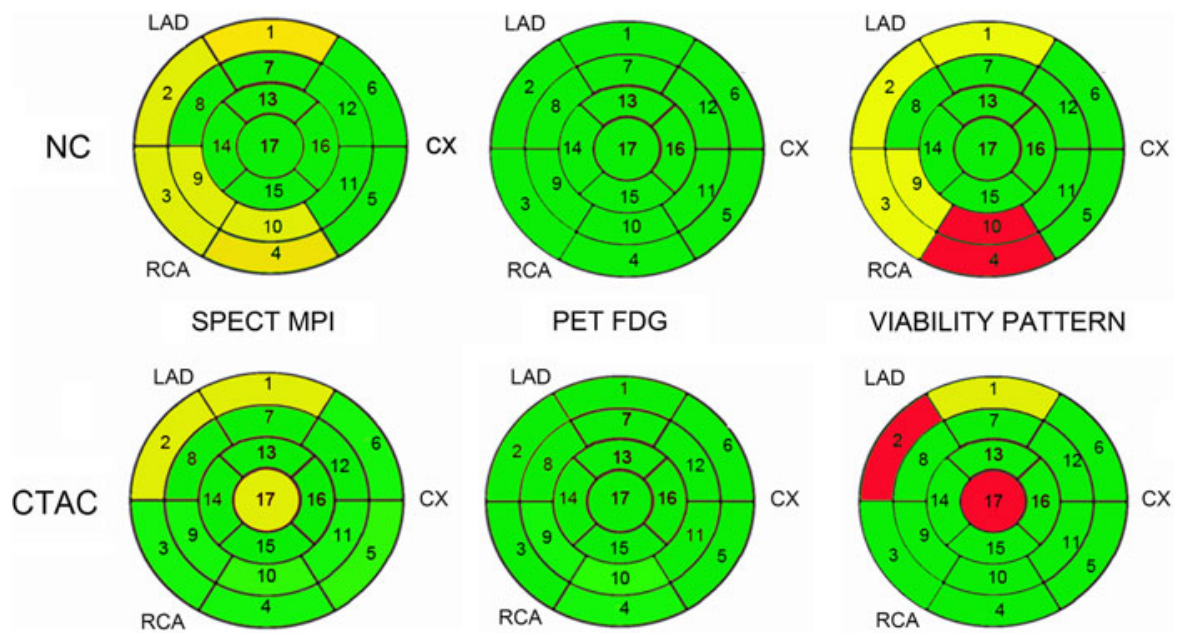

Fig. 3 In this illustrative example there were mild (yellow) perfusion defects on SPECT MPI in the LAD and RCA territory extending from basal anterior and septal to the inferior segments using NC (upper row). After CTAC (lower row) there was no inferior and an additional apical defect was observed. The metabolic uptake on PET FDG was preserved (normal uptake, green) in all segments for both NC and CTAC. The third panel shows the impact of CTAC on the viability pattern: the most striking effect is that the CTAC results in a

Table 3 Viability pattern with no correction of attenuation (NC) and CT attenuation correction (CTAC)

\begin{tabular}{|c|c|c|c|c|}
\hline \multirow{2}{*}{$\begin{array}{l}\text { Territory (\% } \\
\text { of segments) }\end{array}$} & \multicolumn{4}{|c|}{ Patterns of viability } \\
\hline & $\begin{array}{l}\text { Normal } \\
(\%)\end{array}$ & $\begin{array}{l}\text { Mismatch } \\
(\%)\end{array}$ & $\begin{array}{l}\text { Mild match } \\
(\%)\end{array}$ & $\begin{array}{l}\text { Scar } \\
(\%)\end{array}$ \\
\hline \multicolumn{5}{|l|}{ ALL } \\
\hline $\mathrm{NC}$ & 59 & 19 & 16 & 6 \\
\hline CTAC & 71 & 11 & 14 & 4 \\
\hline \multicolumn{5}{|l|}{ LAD } \\
\hline $\mathrm{NC}$ & 66 & 13 & 14 & 7 \\
\hline CTAC & 66 & 14 & 14 & 6 \\
\hline \multicolumn{5}{|l|}{$\mathrm{CX}$} \\
\hline $\mathrm{NC}$ & 74 & 10 & 13 & 3 \\
\hline CTAC & 82 & 5 & 12 & 1 \\
\hline \multicolumn{5}{|l|}{$\mathrm{RCA}$} \\
\hline $\mathrm{NC}$ & 34 & 36 & 21 & 9 \\
\hline CTAC & $66^{*}$ & $13 *$ & 16 & 5 \\
\hline
\end{tabular}

$\overline{A L L \text { all segments, } L A D \text { left anterior descending artery, } C X}$ circumflex artery, $R C A$ right coronary artery, $N C$ no attenuation correction, CTAC CT attenuation correction. $* P<0.001$ versus NC

interpretation of viability based on the flow-metabolism mismatch pattern. This is important because strong evidence exists from a large body of literature decrease in the number of segments with mismatch (yellow) from 4 to 1 . As a consequence, the decision to revascularize would be changed towards conservative treatment. In addition, the allocation of mild match changes from inferior to septal and apical segments. $N C$ no attenuation correction, CTAC CT attenuation correction, MPI myocardial perfusion imaging, LAD territory $=$ segments $1,2,7,8,13,14,17, \mathrm{CX}$ territory $=$ segments $5,6,11,12,16, \mathrm{RCA}$ territory $=$ segments $3,4,9,10,15$

including mainly retrospective [15] but also recent prospective studies [16] that proof of viability prior to revascularization is a major determinant of improved outcome for patients with dysfunctional myocardium in the setting of CAD.

Although there are many different nuclear techniques for viability evaluation including optimized protocols with nitrates enhancement [17, 18], PET FDG has been established as the most sensitive method to discriminate preserved viability from scar. Nevertheless this technique has been hampered by the large variability of viability definition used in different studies. After recognising the importance of standardization by use of oral glucose load or hyperinsulinemic euglycemic clamp, major differences have remained between studies reporting FDGonly versus those corrected for the large individual and regional variations in cardiac glucose utilisation by normalization to perfusion or contractility [19-21].

Normalization of FDG to MPI is the most widely accepted method because it enables the identification of regions with flow impairment but preserved viability, which are the ideal targets for revascularization, while regions of preserved flow are used as reference for normal metabolism in the FDG study. 
SPECT MPI is more widely available than PET MPI and is equally accurate in conjunction with PET FDG for the prediction of functional recovery of dysfunctional segments, symptoms of heart failure and improved survival [19, 20]. Vom Dahl et al. [12] evaluated 161 patients for viability by ${ }^{99 \mathrm{~m}} \mathrm{Tc}$-sestamibi SPECT MPI and PET FDG and noted after revascularization a significant improvement in global and regional left ventricular function predominantly in patients with mismatch on flow-metabolism pattern whereas those with mild-match and scar tissue did mostly not improve. These results were confirmed by other studies using ${ }^{99 \mathrm{~m}}$ Tc-tetrofosmin SPECT emphasizing the prognostic role of combined ${ }^{99 \mathrm{~m}} \mathrm{Tc}$-tetrofosmin SPECT MPI/PET FDG in patients with CAD and chronic dysfunctional left ventricular myocardium [22]. However, SPECT MPI can be affected by soft tissue attenuation which not only impairs accuracy to detect ischemia but also may overestimate the flow-metabolism mismatch when it is used in conjunction with PET FDG for viability assessment.

Recently, CTAC has been established as a tool to improve the accuracy of SPECT MPI for assessing ischemic heart disease by correcting non-uniform attenuation of soft tissue [3, 4]. This improvement in accuracy is to a great proportion due to successful correction of attenuation artifacts, often located in the RCA territory. Similarly, CTAC has been shown to be helpful in discriminating artifacts due to left bundle branch block from true ischemia [23]. After applying CTAC in our study, the proportion of segments with perfusion defects having less than $70 \%$ and less than $50 \%$ uptake decreased by 28 and $49 \%$, respectively. This had a substantial impact on the interpretation of viability pattern, as $25 \%$ of segments originally classified as scar were reclassified and the amount of normal segments increased by $20 \%$. More importantly there was a $42 \%$ decrease in dysfunctional segments with mismatch pattern representing hibernating myocardium. These segments are generally considered to have the greatest potential of functional recovery after revascularisation and therefore qualify for such procedure. This indicates that the use of CTAC for SPECT MPI in conjunction with PET FDG may have a profound impact on the decision towards revascularization.

Because only patients with sufficient amount of viable myocardium benefit from revascularization procedures, accurate assessment of viable tissue but also correct location of the jeopardized myocardium is crucial. Inaccurate viability interpretation may either falsely deny the patient an appropriate revascularization, or expose patients with advanced coronary disease unnecessarily to increased risk of revascularization without predictable benefits [2426]. When taking three viable segments $(18 \%$ of the left ventricle), as threshold to indicate revascularization, the use of CTAC in our study decreased by $54 \%$ the number of patients suitable for revascularization. A threshold to three segments seems reasonable and is supported by previous studies testing viability before revascularization with the endpoint of improved left ventricular ejection fraction [13, 27]. In line with the literature, variability of MPI findings was largest in the inferior segments, most probably due to attenuation artifacts which were successfully corrected by CTAC. Consequently, the most prominent differences in viability interpretations between $\mathrm{NC}$ and CTAC were observed in the RCA territory, where CTAC resulted in a greater number of normal segments while the number of mismatch segments decreased $[3,4]$.

In the CX territory, the incidence of segments with mismatch patterns was unaffected while the specific location of the different viability patterns was subject to large variability after CTAC as reflected by the modest agreement in viability pattern between $\mathrm{NC}$ and CTAC. This may trigger revascularization of different target vessel branches, potentially jeopardizing the clinical benefit of the procedure.

\section{Study limitations}

A limitation of our study is the fact that we did not use glucose-insulin clamp or acipimox as a mean to optimize the quality of FDG uptake. It may also be perceived as a limitation of the present study that we did not assess the impact of CTAC on the prediction of outcome, particularly because most outcome data rely on studies on the pre-AC era. This, however, was beyond the scope of the present study. Nevertheless, as CTAC has repeatedly been shown to improve accuracy of MPI it appears appropriate, although still hypothetic, to assume that this may have the potential to translate into improved viability assessment. This is also supported by small retrospective previous studies using line source based AC before introduction of CTAC $[28,29]$ but remains to be elucidated in 
prospective studies using CTAC. Furthermore, it should be mentioned that information from gated SPECT and from the stress perfusion study were not incorporated into the analysis of viability. Both might have diminished the differences in viability assessment between NC and AC studies, although stress perfusion is not routinely incorporated into myocardial viability assessment. Although one could claim that the present study lacks a firm gold standard, the results from the ICA largely support the findings of CTAC. Finally, the use of CTAC adds radiation exposure to the patient. Compared to the total effective radiation dose from PET FDG $(8 \mathrm{mSV})$ and ${ }^{99 \mathrm{~m}}$ Tc-tetrofosmin SPECT $(10 \mathrm{mSV})$, the contribution of CTAC (1 mSV) accounts for approximately $5 \%$. One single CTAC scan may be used for AC of both SPECT and PET, thus avoiding additional radiation dose.

\section{Clinical implication}

When evaluating patients with dysfunctional myocardium for possible revascularization, the use of CTAC may have a profound impact on the interpretation with regard to presence or absence, location of viable tissue.

\section{Conclusion}

Different interpretation of myocardial viability can be observed when using CTAC instead of NC SPECT MPI as reference for PET FDG scans. Whether this would have an impact on the prognostic accuracy requires further careful prospective investigation.

\section{References}

1. Kaufmann PA, Camici PG (2005) Myocardial blood flow measurement by pet: technical aspects and clinical applications. J Nucl Med 46(1):75-88

2. Kaufmann PA (2007) 82-rubidium-the dawn of cardiac pet in europe? Eur J Nucl Med Mol Imaging 34(12): 1963-1964

3. Fricke E, Fricke H, Weise R, Kammeier A, Hagedorn R, Lotz N, Lindner O, Tschoepe D, Burchert W (2005) Attenuation correction of myocardial spect perfusion images with low-dose ct: evaluation of the method by comparison with perfusion pet. J Nucl Med 46(5):736-744
4. Masood Y, Liu YH, Depuey G, Taillefer R, Araujo LI, Allen S, Delbeke D, Anstett F, Peretz A, Zito MJ, Tsatkin V, Wackers FJ (2005) Clinical validation of spect attenuation correction using X-ray computed tomographyderived attenuation maps: multicenter clinical trial with angiographic correlation. J Nucl Cardiol 12(6):676-686

5. Schepis T, Gaemperli O, Koepfli P, Ruegg C, Burger C, Leschka S, Desbiolles L, Husmann L, Alkadhi H, Kaufmann PA (2007) Use of coronary calcium score scans from stand-alone multislice computed tomography for attenuation correction of myocardial perfusion spect. Eur $\mathbf{J}$ Nucl Med Mol Imaging 34(1):11-19

6. Gaemperli O, Schepis T, Valenta I, Koepfli P, Husmann L, Scheffel H, Leschka S, Eberli FR, Luscher TF, Alkadhi H, Kaufmann PA (2008) Functionally relevant coronary artery disease: comparison of 64-section ct angiography with myocardial perfusion spect. Radiology 248(2):414-423

7. Tillisch J, Brunken R, Marshall R, Schwaiger M, Mandelkern M, Phelps M, Schelbert H (1986) Reversibility of cardiac wall-motion abnormalities predicted by positron tomography. N Engl J Med 314(14):884-888

8. Koepfli P, Hany TF, Wyss CA, Namdar M, Burger C, Konstantinidis AV, Berthold T, Von Schulthess GK, Kaufmann PA (2004) Ct attenuation correction for myocardial perfusion quantification using a pet/ct hybrid scanner. J Nucl Med 45(4):537-542

9. Schepis T, Gaemperli O, Treyer V, Valenta I, Burger C, Koepfli P, Namdar M, Adachi I, Alkadhi H, Kaufmann PA (2007) Absolute quantification of myocardial blood flow with 13n-ammonia and 3-dimensional pet. J Nucl Med 48(11):1783-1789

10. Cerqueira MD, Weissman NJ, Dilsizian V, Jacobs AK, Kaul S, Laskey WK, Pennell DJ, Rumberger JA, Ryan T, Verani MS (2002) Standardized myocardial segmentation and nomenclature for tomographic imaging of the heart. A statement for healthcare professionals from the cardiac imaging committee of the council on clinical cardiology of the American heart association. Int J Cardiovasc Imaging 18(1):539-542

11. Hesse B, Tagil K, Cuocolo A, Anagnostopoulos C, Bardies M, Bax J, Bengel F, Busemann Sokole E, Davies G, Dondi M, Edenbrandt L, Franken P, Kjaer A, Knuuti J, Lassmann M, Ljungberg M, Marcassa C, Marie PY, McKiddie F, O'Connor M, Prvulovich E, Underwood R, van Eck-Smit B (2005) Eanm/esc procedural guidelines for myocardial perfusion imaging in nuclear cardiology. Eur J Nucl Med Mol Imaging 32(7):855-897

12. vom Dahl J, Altehoefer C, Sheehan FH, Buechin P, Schulz G, Schwarz ER, Koch KC, Uebis R, Messmer BJ, Buell U, Hanrath P (1997) Effect of myocardial viability assessed by technetium- $99 \mathrm{~m}$-sestamibi spect and fluorine-18-fdg pet on clinical outcome in coronary artery disease. J Nucl Med 38(5):742-748

13. Schinkel AF, Poldermans D, Elhendy A, Bax JJ (2007) Assessment of myocardial viability in patients with heart failure. J Nucl Med 48(7):1135-1146

14. Rentrop KP, Cohen M, Blanke H, Phillips RA (1985) Changes in collateral channel filling immediately after controlled coronary artery occlusion by an angioplasty balloon in human subjects. J Am Coll Cardiol 5(3): 587-592 
15. Underwood SR, Bax JJ, vom Dahl J, Henein MY, Knuuti J, van Rossum AC, Schwarz ER, Vanoverschelde JL, van der Wall EE, Wijns W (2004) Imaging techniques for the assessment of myocardial hibernation. Report of a study group of the european society of cardiology. Eur Heart $\mathbf{J}$ 25(10):815-836

16. Beanlands RS, Nichol G, Huszti E, Humen D, Racine N, Freeman M, Gulenchyn KY, Garrard L, deKemp R, Guo A, Ruddy TD, Benard F, Lamy A, Iwanochko RM (2007) F-18-fluorodeoxyglucose positron emission tomography imaging-assisted management of patients with severe left ventricular dysfunction and suspected coronary disease: a randomized, controlled trial (parr-2). J Am Coll Cardiol 50(20):2002-2012

17. Iskandrian AS, Hakki AH, Kane SA, Goel IP, Mundth ED, Segal BL (1983) Rest and redistribution thallium-201 myocardial scintigraphy to predict improvement in left ventricular function after coronary arterial bypass grafting. Am J Cardiol 51(8):1312-1316

18. Galli M, Marcassa C, Imparato A, Campini R, Orrego PS, Giannuzzi P (1994) Effects of nitroglycerin by technetium$99 \mathrm{~m}$ sestamibi tomoscintigraphy on resting regional myocardial hypoperfusion in stable patients with healed myocardial infarction. Am J Cardiol 74(9):843-848

19. Allman KC, Shaw LJ, Hachamovitch R, Udelson JE (2002) Myocardial viability testing and impact of revascularization on prognosis in patients with coronary artery disease and left ventricular dysfunction: a meta-analysis. J Am Coll Cardiol 39(7):1151-1158

20. Rizzello V, Poldermans D, Biagini E, Schinkel AF, Boersma E, Boccanelli A, Marwick T, Roelandt JR, Bax JJ (2009) Prognosis of patients with ischaemic cardiomyopathy after coronary revascularisation: relation to viability and improvement in left ventricular ejection fraction. Heart 95(15):1273-1277

21. Gerber BL, Ordoubadi FF, Wijns W, Vanoverschelde JL, Knuuti MJ, Janier M, Melon P, Blanksma PK, Bol A, Bax JJ, Melin JA, Camici PG (2001) Positron emission tomography using(18)f-fluoro-deoxyglucose and euglycaemic hyperinsulinaemic glucose clamp: optimal criteria for the prediction of recovery of post-ischaemic left ventricular dysfunction. Results from the European community concerted action multicenter study on use of (18)f-fluoro-deoxyglucose positron emission tomography for the detection of myocardial viability. Eur Heart $\mathbf{J}$ 22(18):1691-1701

22. Beanlands RS, Hendry PJ, Masters RG, deKemp RA, Woodend K, Ruddy TD (1998) Delay in revascularization is associated with increased mortality rate in patients with severe left ventricular dysfunction and viable myocardium on fluorine 18-fluorodeoxyglucose positron emission tomography imaging. Circulation 98(19(Suppl)):II51-II56

23. Hoefflinghaus T, Husmann L, Valenta I, Moonen C, Gaemperli O, Schepis T, Namdar M, Koepfli P, Siegrist PT, Kaufmann PA (2008) Role of attenuation correction to discriminate defects caused by left bundle branch block versus coronary stenosis in single photon emission computed tomography myocardial perfusion imaging. Clin Nucl Med 33(11):748-751

24. Fox K, Garcia MA, Ardissino D, Buszman P, Camici PG, Crea F, Daly C, De Backer G, Hjemdahl P, Lopez-Sendon J, Marco J, Morais J, Pepper J, Sechtem U, Simoons M, Thygesen K, Priori SG, Blanc JJ, Budaj A, Camm J, Dean V, Deckers J, Dickstein K, Lekakis J, McGregor K, Metra M, Osterspey A, Tamargo J, Zamorano JL (2006) Guidelines on the management of stable angina pectoris: executive summary: the task force on the management of stable angina pectoris of the European society of cardiology. Eur Heart J 27(11):1341-1381

25. Patel MR, Dehmer GJ, Hirshfeld JW, Smith PK, Spertus JA (2009) Accf/scai/sts/aats/aha/asnc 2009 appropriateness criteria for coronary revascularization: a report by the american college of cardiology foundation appropriateness criteria task force, society for cardiovascular angiography and interventions, society of thoracic surgeons, american association for thoracic surgery, american heart association, and the american society of nuclear cardiology endorsed by the american society of echocardiography, the heart failure society of America, and the society of cardiovascular computed tomography. J Am Coll Cardiol 53(6):530-553

26. Baker DW, Jones R, Hodges J, Massie BM, Konstam MA, Rose EA (1994) Management of heart failure: III. The role of revascularization in the treatment of patients with moderate or severe left ventricular systolic dysfunction. JAMA 272(19):1528-1534

27. Chareonthaitawee P, Gersh BJ, Araoz PA, Gibbons RJ (2005) Revascularization in severe left ventricular dysfunction: the role of viability testing. J Am Coll Cardiol 46(4):567-574

28. Slart RH, Bax JJ, Sluiter WJ, van Veldhuisen DJ, Jager PL (2004) Added value of attenuation-corrected tc-99m tetrofosmin spect for the detection of myocardial viability: comparison with fdg spect. J Nucl Cardiol 11(6):689-696

29. Matsunari I, Boning G, Ziegler SI, Nekolla SG, Stollfuss JC, Kosa I, Ficaro EP, Schwaiger M (1998) Attenuationcorrected 99mtc-tetrofosmin single-photon emission computed tomography in the detection of viable myocardium: comparison with positron emission tomography using 18ffluorodeoxyglucose. J Am Coll Cardiol 32(4):927-935 